\title{
Phenotype-Genotype Correlations and
}

\section{Distribution of Key Virulence Factors in}

\section{Enterococcus faecalis Isolated from Patients with Urinary Tract Infections}

\author{
Yomna A Hashem (D) \\ Khaled A Abdelrahman (D) \\ Ramy K Aziz (iD) 2,3 \\ 'Department of Microbiology, Faculty of \\ Pharmacy, The British University in Egypt, \\ Cairo, Egypt; ${ }^{2}$ Department of \\ Microbiology and Immunology, Faculty of \\ Pharmacy, Cairo University, Cairo, Egypt; \\ ${ }^{3}$ Microbiology and Immunology Research \\ Program, Children's Cancer Hospital \\ Egypt, 57357, Cairo, Egypt
}

Background and Objective: Enterococcus faecalis can cause different nosocomial infections, especially urinary tract infection (UTI). Pathogenicity of E. faecalis is driven by various virulence factors; however, no specific genetic pattern is restricted to a particular type of infection. The current study aimed to investigate the correlation between different virulence factors in E. faecalis clinical isolates causing UTIs.

Methods: We phenotypically analyzed 60 urinary isolates, identified as E. faecalis, for biofilm formation, gelatinase, protease and hemolytic activities by Crystal Violet assay, gelatin hydrolysis, casein hydrolysis and blood agar hemolysis assays, respectively. Additionally, we detected different genes associated with species identification, virulence phenotypes, adherence and quorum sensing by the polymerase chain reaction (PCR). The detected genes included D-alanine-D-alanine ligase $(d d l)$, cytolysin $(c y l)$, gelatinase ( $g e l E)$, serine protease $(s p r E)$, faecal streptococci regulator locus genes $\left(f_{s r} A, f_{s r} B, f_{s r} C\right)$, pili (pil), adhesin to collagen of E. faecalis (ace) and aggregation substance (agg).

Results: All isolates formed biofilms, mostly with strong to moderate ability. Although gelE was detected in $87 \%$ of the isolates, only $22 \%$ of the isolates had gelatinase activity. Similar phenotype-genotype incongruities were observed with hemolysis and casein hydrolysis activities, as the isolates that expressed these two phenotypes were fewer than those carrying the genes encoding them.

Conclusion: A clear variability in virulence gene distribution among the isolates was observed, and no particular pattern was associated with UTI. Whereas all isolates carried at least ace and pil, whose products are involved in adherence, which is a virulence phenotype that is required for urinary colonization, six isolates carried the entire set of investigated genes. Statistical analysis of the results suggests $c y l$ as a biomarker for hemolytic activity, $f_{s r} B$ as a diagnostic biomarker for the gelatinase activity, and gelE-sprE as predictors for biofilm formation strength in E. faecalis.

Keywords: enterococci, urinary tract infections, biofilm formation, gelatinase, quorum sensing, statistical association

\section{Introduction}

Correspondence: Ramy K Aziz Department of Microbiology and Immunology, Faculty of Pharmacy, Cairo University, Cairo, II 562, Egypt

Tel +201007I58450

Email ramy.aziz@gmail.com
Enterococci are Gram-positive, facultative anaerobic organisms that can be seen under the microscope as single, pairs or short chains of cocci. ${ }^{1}$ Although they are members of the gastrointestinal microbiota, ${ }^{2}$ enterococci are opportunistic 
pathogens, with several strains capable of causing community- and hospital-acquired infections, notably in immunocompromised hosts. Infections caused by enterococci include endocarditis, bacteremia and urinary tract infections (UTIs). ${ }^{3}$ Among many species identified, E. faecalis and E. faecium are the most common species capable of causing infection and posing a threat of antimicrobial resistance, with $E$. faecalis accounting for the majority of infections. $^{4,5}$

The first crucial step in E. faecalis pathogenesis is the adherence to host tissues, especially UTIs. ${ }^{4}$ Virulence factors associated with adherence include an aggregation substance (Agg), a surface protein expressed in response to pheromone induction that mediates the adherence of E. faecalis to renal epithelial cells. ${ }^{6}$ Another surface protein that facilitates the bacterial adherence to collagen is the adhesin to collagen of E. faecalis (Ace). ${ }^{7}$ Both Agg and Ace play a critical role in adherence to and colonization of host tissues. ${ }^{8}$

E. faecalis is also capable of attaching irreversibly to biotic and abiotic surfaces, forming biofilms. ${ }^{9}$ These biofilms were shown to confer additional antibiotic resistance to the bacteria, as opposed to planktonic cells, which are more exposed and vulnerable to antibiotic therapy. ${ }^{10}$

In addition to adherence, E. faecalis secrete virulence factors that contribute to the severity of their infection. Cytolysin, a secreted toxin expressed in response to pheromones, contributes to the pathogenicity of E. faecalis by causing blood hemolysis. ${ }^{11}$

A gelatinase enzyme (GelE), as well as a serine protease (SprE), hydrolyze gelatin and casein, respectively. ${ }^{12}$ The ability of gelatinase to damage host tissues plays an important role in spreading of enterococci in their host. ${ }^{13}$ Gelatinase is also important for biofilm formation. Hancock and Perego demonstrated that gelatinase promotes the aggregation of the cells in microcolonies, which constitutes the initial step of biofilm formation. ${ }^{14}$

A major quorum sensing system in E. faecalis, the Fsr regulator locus, is encoded by $f s r A, f s r B$ and $f s r C$ genes, which regulate the expression of both gelatinase and serine protease. $^{15}$ The Fsr quorum-sensing system controls biofilm development through regulating the production of gelatinase. $^{14}$

E. faecalis is now emerging as a serious cause of both hospital- and community-acquired UTIs, ${ }^{16,17}$ which can lead to serious, fatal complications like bacteremia. ${ }^{16}$ While no particular set of genes has been proposed to be associated with UTIs, no systematic investigation of phenotype-genotype concordance has been established for this clinically important type of infection. Guided by a prior study ${ }^{18}$ on enterococci isolated from various tissues, we launched the current study to phenotypically and genotypically investigate and correlate virulence factors, such as biofilm formation, gelatin and casein hydrolysis, as well as blood hemolysis, in E. faecalis clinical isolates from patients with community-acquired UTIs in Egypt.

\section{Materials and Methods}

\section{Bacterial Isolates and Culture Media}

Sixty Enterococcus faecalis isolates were obtained from Egyptian clinical laboratories in the period from 2018 to 2019, and they had been pre-collected from nonhospitalized patients with community-acquired UTIs and biobanked afterwards.

The bacteria were isolated by the streak-plate method on Enterococcosel agar (BD, USA). Identification to genus level was performed by Gram stain, and biochemical tests, eg, catalase and $6.5 \% \mathrm{NaCl}$ tolerance tests. Surface streaking of the isolates on Chromogenic UTI agar (Oxoid, UK) was also confirmatory of enterococcal identity. Identification to species level was done by PCR with specific primers amplifying the $d d l$ gene of E. faecalis ATCC29212 was used as reference strain (positive control).

\section{Phenotypic Detection of Virulence Factors Biofilm Assay}

Biofilm formation was assessed by the Crystal Violet assay according to well-established methods, ${ }^{19}$ with some modifications. Trypticase Soy Broth (TSB from Oxoid, UK), containing $0.5 \%$ glucose, was inoculated with the overnight cultures, and the culture density was adjusted to be equivalent to 0.5 McFarland by spectrophotometry (Unicam, UK). Cultures with the adjusted concentrations was diluted again with TSB containing $0.5 \%$ glucose. $^{20}$ Sterile flat-bottom 96 well microtiter plates (Greiner CELLSTAR) were inoculated with $200 \mu \mathrm{L}$ of the diluted cultures aseptically, and each isolate was added in triplicate wells. Negative controls of TSB containing $0.5 \%$ glucose alone were also included. The contents of the plates were discarded after overnight incubation, and the wells were washed with saline three times and left to dry. Methanol was used to fix the adherent cells. Fixed adherent cells were stained with 1\% (W/V) Crystal Violet for 15 minutes; excess stain was removed by washing; and plates 
were left to dry. Crystal Violet bound with the adherent cells was re-dissolved in glacial acetic acid. The optical density (OD) was measured at $545 \mathrm{~nm}$ in a plate reader (Biotek, USA), and the median of three readings was taken. The strength of biofilm was classified according to the OD readings (Table 1$)^{21}$

\section{Gelatinase Activity}

The gelatinase activity of each isolate was determined by the method of Su and colleagues, ${ }^{22}$ with the following modification: Gelatin media was prepared by the addition of 3\% gelatin powder (HiMedia, India) to Brain Heart Infusion (BHI) agar (Oxoid, UK). Isolates were streaked on gelatin plates, incubated at $37^{\circ} \mathrm{C}$, and, after incubation, Frazier solution was added to the plates. The formation of transparent halo zone around the colonies upon addition of Frazier solution indicated the presence of gelatinase activity. ${ }^{18,22,23}$

\section{Protease Activity}

The protease activity of the isolates was assessed by the casein hydrolysis assay. Casein hydrolysis was evaluated by cultivation of the isolates on BHI agar containing 1.5\% (w/v) skimmed milk. The formation of a transparent zone around the colonies after incubation indicated protease activity. ${ }^{24,25}$

\section{Hemolytic Activity}

The hemolytic activity was assessed by cultivation of the isolates on blood agar base (Oxoid, UK) supplemented with 5\% defibrinated blood. A clear zone around the colonies, observed after 24 -hour incubation at $37^{\circ} \mathrm{C}$, indicated hemolytic activity. ${ }^{26,27}$

\section{Genotypic Detection of Virulence Factors}

Screening for $d d l$ gene for species identification ${ }^{28}$ and virulence genes, including gelE, sprE, fsr $A, f_{s r} B, f_{s r} C$, pil, ace, agg, and cyl, was carried out by PCR, as previously detailed. ${ }^{18}$ Bacterial DNA was extracted from each isolate by boiling a few colonies in TRISEDTA buffer. Reaction mixtures consisted of $0.25 \mu \mathrm{g}$ extracted DNA, $1.5 \mathrm{mM} \mathrm{MgCl}_{2}, 10 \mathrm{pM}$ of each primer (Table 2), $200 \mu \mathrm{M}$ of each deoxyribonucleotide and 0.5 U Taq polymerase (Qiagen, Germany). ${ }^{29}$ PCR amplification was performed in a SensoQuest (Germany) thermocycler. The amplification conditions were: an initial denaturation step at $95{ }^{\circ} \mathrm{C}$ for 2 minutes, followed by 30 cycles of denaturation at $95^{\circ} \mathrm{C}$ for 30 seconds, annealing for 30 seconds at different temperatures according to the primers used, extension at $72^{\circ} \mathrm{C}$ for 30 seconds. The reaction was concluded by a final extension step at $72{ }^{\circ} \mathrm{C}$ for 5 minutes. PCR products were analyzed by gel electrophoresis and visualized under UV light. ${ }^{18}$

\section{Statistical Analysis}

Parametric data were analyzed for significance by Student's $t$-test, and $p$ values $\leq 0.05$ were considered statistically significant. Non-parametric data were analyzed by Kruskal-Wallis test or Wilcoxon test, depending on the number of variables. Correlations, Chi-square, and Fisher's Exact tests were performed in R (URL: https:// www.r-project.org/). In most instances, presence and absence values were converted into pseudonumeric variables (with 1 and 0 ) to compute Pearson correlation coefficients and create a correlation matrix. Specific $\mathrm{R}$ packages that were used in statistical analysis or data visualization are corrplot and beanplot.

\section{Results}

\section{Bacterial Isolates}

The 60 isolates identified as E. faecalis showed characteristic brownish-black colonies, when streaked on Enterococcosel agar, and blue colonies upon cultivation on Chromogenic UTI agar.

Table I Classification of Biofilm Strength According to O.D. Measurements

\begin{tabular}{|c|c|c|}
\hline Cut-off Value Calculation & Median ${ }^{b}$ of O.D. Values & Biofilm Strength \\
\hline O.D. $\leq$ O.D. $\mathrm{c}^{\mathrm{a}}$ & O.D. $\leq 0.065$ & None \\
\hline O.D.c < O.D. $\leq 2 \times$ O.D.c & $0.065<$ O.D. $\leq 0.13$ & Weak \\
\hline $2 \times$ O.D.c $<$ O.D. $\leq 4 \times$ O.D.c & $0.13<$ O.D. $\leq 0.26$ & Moderate \\
\hline O.D. $>4 \times$ O.D.c & $O D>0.26$ & Strong \\
\hline
\end{tabular}

Notes: ${ }^{\mathrm{a} O . D . c}$ is the O.D. of the negative control; ${ }^{\mathrm{b}}$ Because the assay was performed in triplicates, and because some cases had outliers, the median-rather than the mean - was used; however, the biofilm strength classification was not affected by whether the mean or median was used, except in one sample, EU42, whose assay triplicates were on both side of the moderate-strong threshold (Figure IA), and had the highest coefficient of variation (69\%). 
Table 2 List of Primers Pairs Used in the Current Study

\begin{tabular}{|c|c|c|c|c|}
\hline Gene & Forward Primer & Reverse Primer & Product Size (bp) & Reference \\
\hline$d d l$ & ATCAAGTACAGTTAGTCTTTA & AACGATTCAAAGCTAACT & 942 & [28] \\
\hline agg & TCTTGGACACGACCCATGAT & AGAAAGAACATCACCACGAGC & 413 & [18] \\
\hline fsrA & CGTTCCGTCTCTCATAGTTA & GCAGGATTTGAGGTTGCTAA & 474 & [18] \\
\hline fsrB & TAATCTAGGCTTAGTTCCCAC & CTAAATGGCTCTGTCGTCTAG & 428 & [18] \\
\hline fsrC & GTGTTTTTGATTTCGCCAGAGA & TATAACAATCCCCAACCGTG & 716 & [18] \\
\hline gelE & GGTGAAGAAGTTACTCTGAC & GGTATTGAGTTATGAGGGGC & 704 & [18] \\
\hline sprE & CTGAGGACAGAAGACAAGAAG & GGTTTTTCTCACCTGGATAG & 432 & [18] \\
\hline ace & GAATGACCGAGAACGATGGC & CTTGATGTTGGCCTGCTTCC & 615 & [18] \\
\hline pil & GAAGAAACCAAAGCACCTAC & CTACCTAAGAAAAGAAACGCG & 620 & [18] \\
\hline cyl & TGGCGGTATTTTTACTGGAG & TGAATCGCTCCATTTCTTC & 186 & This study \\
\hline
\end{tabular}

\section{Diversity of Virulence Phenotypes Among Enterococcal Isolates}

The Crystal Violet assay showed that all the isolates formed biofilm, but with different strengths. Out of the 60 isolates evaluated for biofilm formation capacity (Figure 1A), 37 isolates (62\%) could form strong biofilm; 15 isolates $(25 \%)$ formed moderate biofilm and eight isolates (13\%) formed weak biofilm (Figure 1B and C). One isolate (designated as Eu42) was excluded from downstream analysis because its biofilm assay readings had a larger spread, spanning the moderate/strong threshold (Figure 1A).

Phenotypic detection of gelatinase activity indicated that 13 isolates were gelatinase positive $(22 \%)$. Protease activity, evaluated by casein hydrolysis, revealed that 34 of the isolates were protease positive $(57 \%)$. Hemolytic activity, detected by complete hemolysis of blood agar (Beta hemolysis), showed that 20 isolates (33\%) were hemolytic (Table S1, Figure 2A and B).

\section{Distribution of Virulence Genes Among Isolates}

Molecular screening for a set of nine virulence genes, encoding different virulence phenotypes, revealed that ace and pil were present in all the isolates, gelE and sprE in $87 \%$, agg in $67 \%$, cyl in $45 \%, f_{s r} A$ in $42 \%, f_{s r} B$ in $28 \%$ and $f s r C$ in $48 \%$ of the isolates (Table S1 and Figure 2). In other words, isolates harbored at least two of the screened genes, with the majority harboring between five and seven genes, while six isolates harbored all nine screened genes (Table S1 and Figure 2A).

Combining the detected phenotypes and the selected set of corresponding genotypes allowed dividing the isolates into different patterns or clusters (Figure 2A), highlighting their diversity and demonstrating the well- established notion in molecular pathogenesis that genotypically diverse isolates may cause similar phenotypes and lead to similar diseases.

\section{Phenotype-Genotype Correlations}

To investigate phenotype-genotype correlations among the isolates, at different levels, we statistically analyzed all screened phenotypes and genotypes against one another, with different tests for associations [Chi-square (Table S2) and Fisher's Exact test (Table S3) for dependence among count data, as well as Pearson's correlation analysis of the presence/absence data of all assays (Figure 3)].

Among the interesting investigated associations were those between virulence phenotypes and the genes known to encode them. The strongest phenotype-genotype congruence was found between hemolytic activity and the cytolysin-encoding $c y l$ gene. Even though only 20 out of $27 \mathrm{cyl}$ positive isolates were hemolytic (Table 3 ), a strong statistically significant dependence was found between hemolysis and cyl (Chi-square $p$-value $=7 \times 10^{-9}$ and Fisher's Test $p$-value $\left.=2 \times 10^{-10}\right)$. The correlation coefficient between hemolysis and $c y l$ was 0.78 (Figure 3).

Biofilm strength was slightly correlated with the sprE and gelE genes (Pearson's coefficient $=0.34$, Figure 3), and their association was statistically significant (Chi-square $p=0.0053$; Fisher's exact test $p=0.011$ ). Association between gelatinase activity and $f_{s r} A, f_{s r} B$ and $f_{s r} C$ was statistically significant (Chi-square $p=0.00001, p=5 \times 10^{-8}$ and $p=0.0011$, respectively, Table 4), but the strongest predictor of this activity was the detection of both $f_{s r} A$ and $f_{s r} B$ in a given isolate (Chi-square $p=2 \times 10^{-8}$ and a correlation coefficient of 0.73 , Figure 3 ). Association between protease activity (caseinase assay) and sprE and gelE was significant (Chi-square $p=0.0201$ ), and the association between blood hemolysis and 
A Biofilm-forming Ability of Isolates

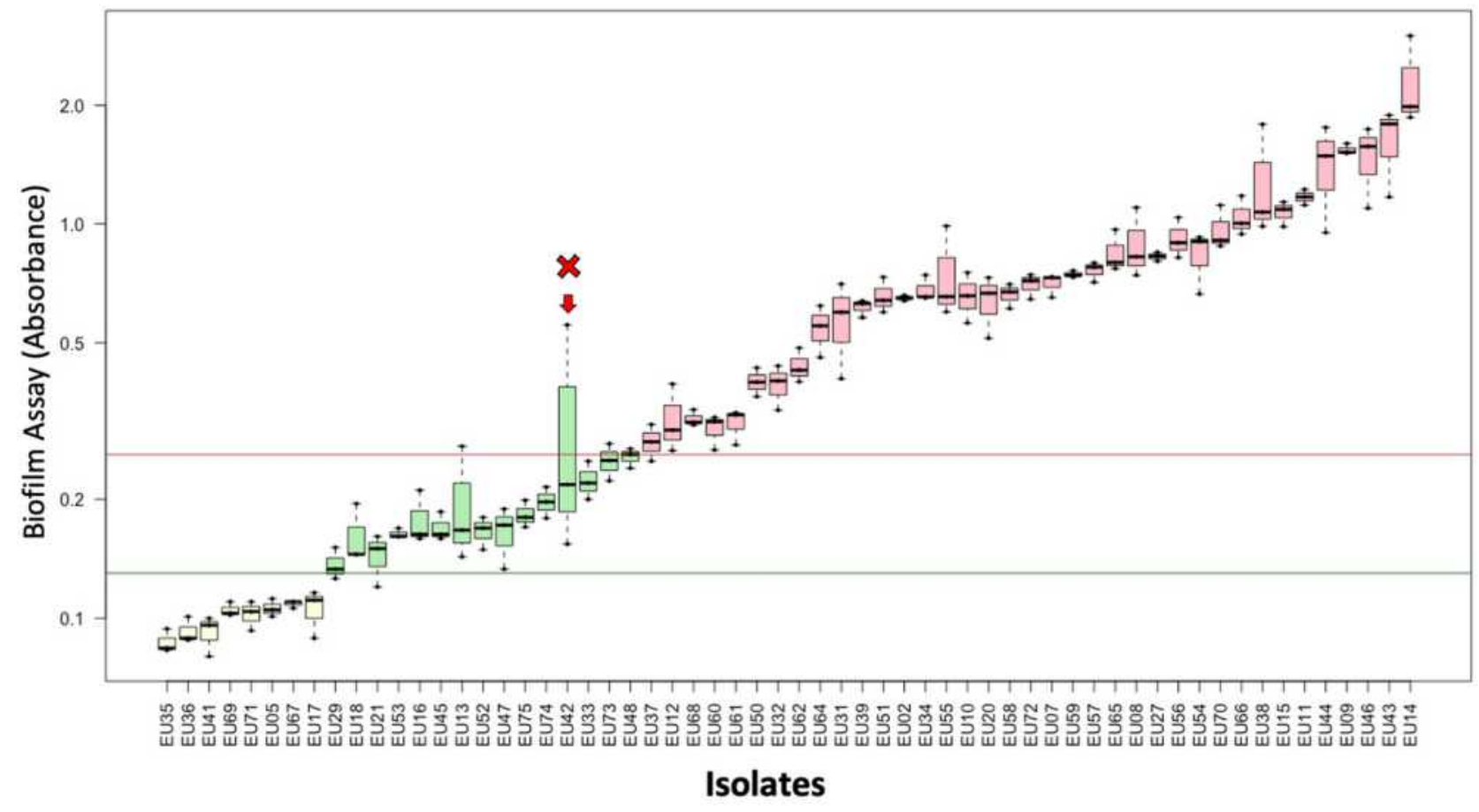

B

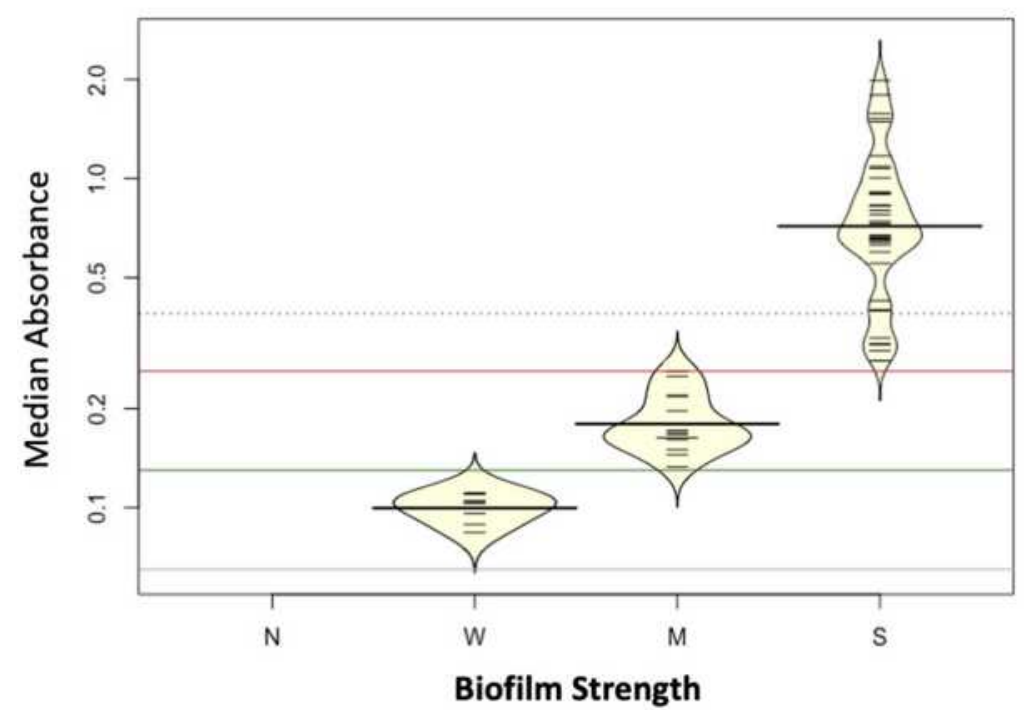

C Biofilm Strength

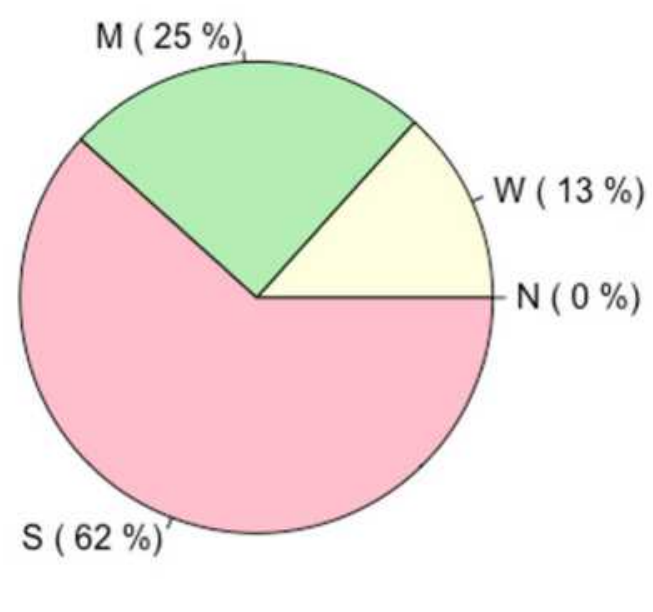

Figure I Biofilm-forming activity of all enterococcal isolates. (A) Boxplots showing the results of the Crystal Violet assay, measured as OD in triplicates. The $Y$ axis represents the actual optical density of each reading, on a log scale. Isolate "EU42" is indicated by an arrow because its interquartile range spans the moderate-strong biofilm-formation threshold, which led to its exclusion from further analysis. (B) Beanplots indicating the distinction of the isolates according to their biofilm assay into weak, moderate, and strong-biofilm formers. Horizontal lines in (A and B) represent the thresholds for biofilm strength classification (Table I): brick red (threshold for "strong" designation; dark green: threshold for "moderate" designation; gray: threshold for "weak" designation. (C) Pie chart for the proportion of isolates with different strengths of biofilm formation.

agg was marginally significant (Fisher's Exact test $p=$ 0.0436, while Chi-square $p=0.0658$ ).

Overall, the correlation between all possible pairs of biologically significant phenotypes and biomarker genes (detected by PCR) was represented as a correlation plot, highlighting the above significant associations, as well as others that are more subtle (Figure 3). 


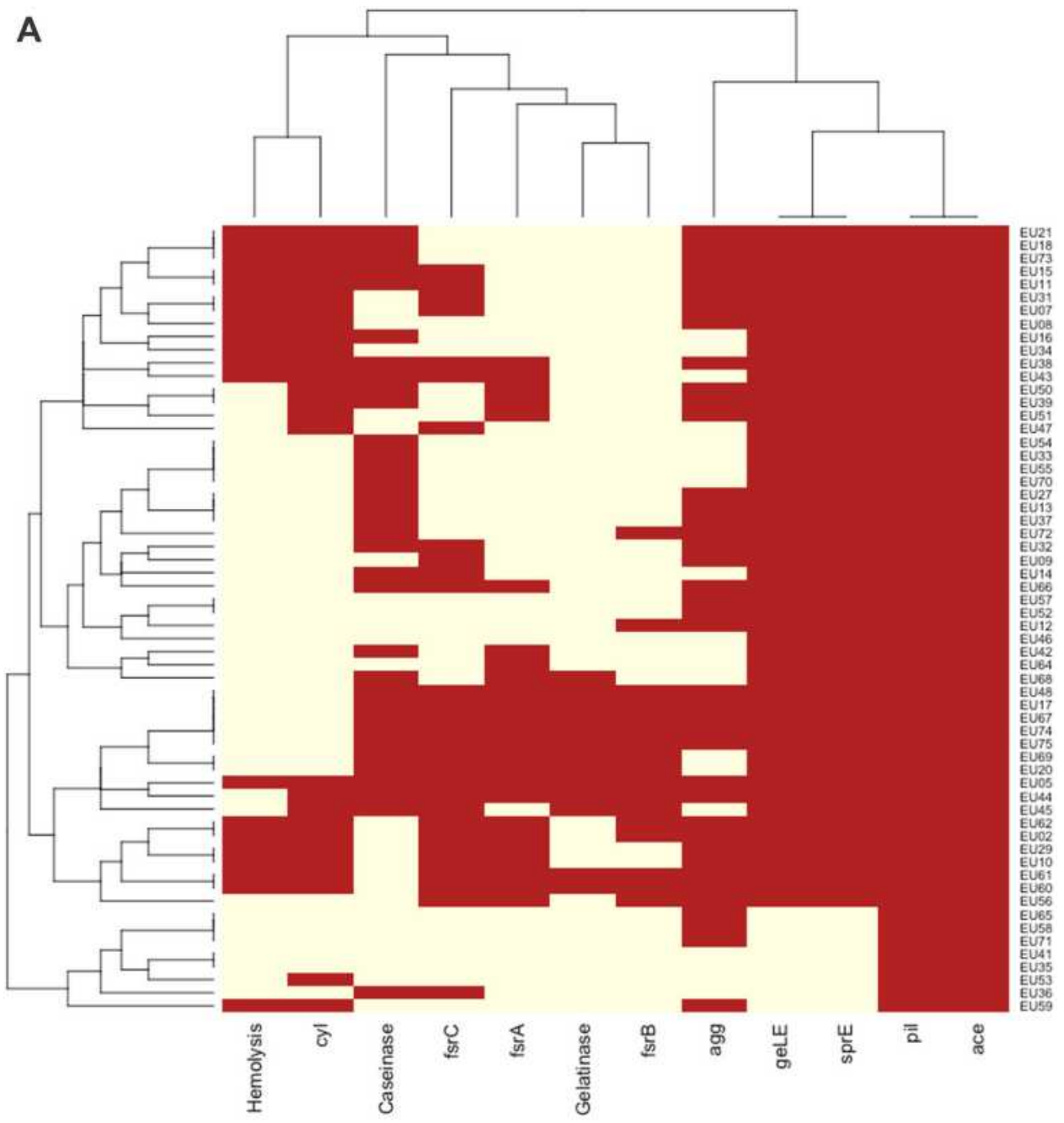

B

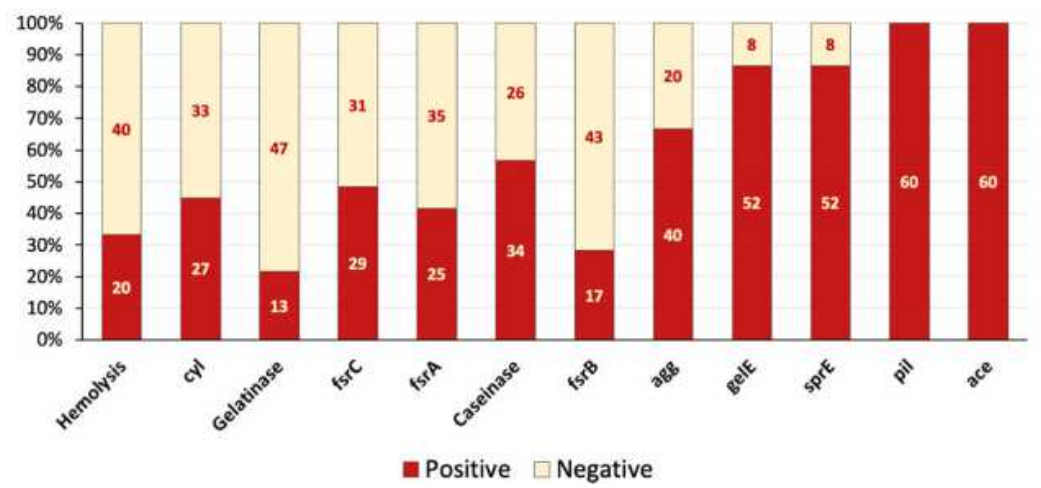

Figure 2 Distribution of screened virulence phenotypes and genotypes among the isolates. (A) Clustering of the isolate and different phenotypic and genotypic markers. Brick red: present (positive assay or PCR-detected gene); light yellow: negative assay or (PCR-negative gene). (B) A stacked bar plot indicating the numbers and proportion of positive and negative virulence phenotypes and genotypes. The measured virulence factors are in the order of clustering in IA.

\section{Discussion}

Enterococci, especially E. faecalis, are important nosocomial pathogens and are among the leading causes of antimicrobial-resistant UTI. ${ }^{1,30}$ The pathogenicity of
E. faecalis is associated with different virulence factors that facilitate adherence and invasiveness. ${ }^{2}$ The aim of the current study was to evaluate the presence of different virulence factor genes, as well as their encoded 


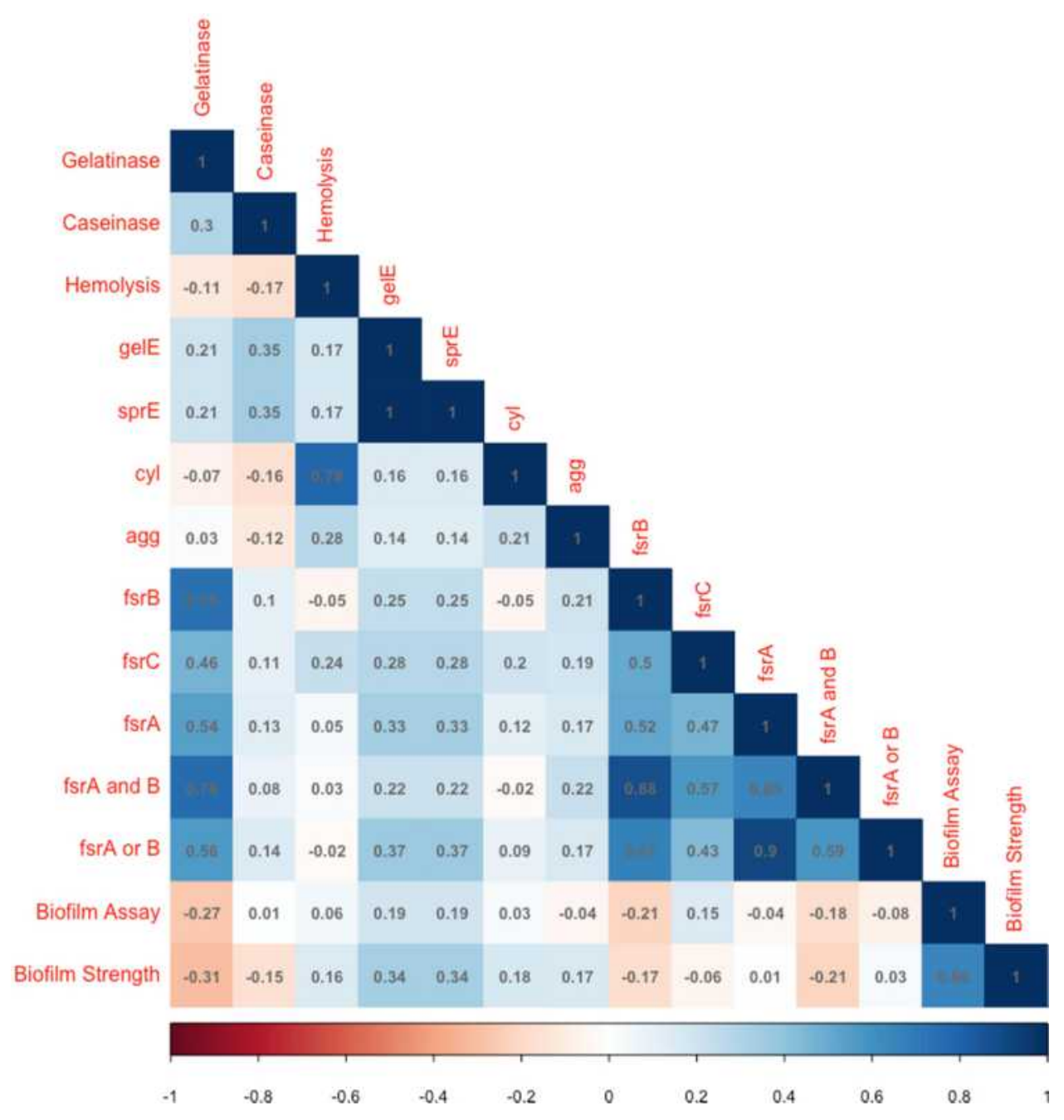

Figure 3 A correlation matrix of major phenotypes and selected set of genotypes measured in this study. The color represents Pearson's correlation coefficient, and its intensity represents the coefficient's value (Shades of blue are positive correlations and shades of orange-brown are negative correlations).

phenotypes, among community-acquired enterococcal clinical isolates causing UTI and to predict the possible correlations between them.

The Identification of enterococci to the species level is important for establishing such correlation, for determining their antimicrobial susceptibility, and consequently for providing the proper treatment. ${ }^{1}$ To confirm the identity of E. faecalis, we resorted to a PCR assay detecting internal fragments of the gene encoding D-alanine-D-alanine ligase, $d d l$, as this gene was described to be diagnostic and to differentiate the two major clinically important species E. faecalis and E. faecium. ${ }^{28}$

Biofilms are key factors for the pathogenicity of UTIs, since they enhance the crucial step of adherence, which protects the bacteria from being flushed by urine. ${ }^{31}$ In the present study, all the isolates were capable of forming a biofilm, and most of them had the ability to form strong to moderate biofilms. This result is in agreement with the work of Seno et al who found that all the enterococcal isolates collected from patients with UTI in Okayama University Hospital could form biofilm. ${ }^{32}$ The high frequency of biofilm formation is also consistent with other studies. ${ }^{33-36}$

In the current study, biofilm formation strength was significantly associated with the presence of gelE and sprE genes. Hancock and Perego, ${ }^{14}$ as well as others, ${ }^{33,37}$ reported earlier the importance of gelE for biofilm formation. On the other hand, Kafil and Mobare ${ }^{30}$ and Kart and Kuştimur $^{38}$ concluded that gelE gene presence had no effect on biofilm formation.

In the current study, the gelE gene was present in $87 \%$ of the isolates. High frequency of gelE gene in enterococcal isolates from UTI was also reported in the other studies. $^{30,39,40}$ Despite this high frequency of detection of gelE, only $22 \%$ of the isolates expressed a phenotypic 
Table 3 Genotype-Phenotype Correlations

\begin{tabular}{|c|c|c|c|c|c|c|}
\hline \multicolumn{7}{|c|}{ Genes Significantly Associated with Hemolytic Activity } \\
\hline $\begin{array}{l}\text { Genotype } \\
\text { Phenotype }\end{array}$ & $c y l+$ & cyl- & Total & $\operatorname{agg}+$ & $\operatorname{agg}-$ & Total \\
\hline Hemolytic activity + & 20 & 0 & 20 & 17 & 3 & 20 \\
\hline Hemolytic activity - & 7 & 33 & 40 & 23 & 17 & 40 \\
\hline \multirow[t]{3}{*}{ Total } & 27 & 33 & 60 & 40 & 20 & 60 \\
\hline & \multicolumn{3}{|c|}{ Chi-square $p=7 \times 10^{-9}$} & \multicolumn{3}{|c|}{ Chi-square $p=0.0658$} \\
\hline & \multicolumn{3}{|c|}{ Fisher's Exact test $p=2 \times 10^{-10}$} & \multicolumn{3}{|c|}{ Fisher's Exact test $p=0.0436$} \\
\hline \multicolumn{7}{|c|}{ Phenotypes Associated withgelE/sprE } \\
\hline $\begin{array}{l}\text { Genotype } \\
\text { Phenotype }\end{array}$ & gelE + & gelE - & Total & sprE + & sprE - & Total \\
\hline \multicolumn{7}{|l|}{ Biofilm strength } \\
\hline Strong & 35 & 3 & 37 & 34 & 3 & 37 \\
\hline Moderate & 12 & I & 14 & 12 & $\mathrm{I}$ & 14 \\
\hline Weak & 5 & 4 & 9 & 4 & 4 & 9 \\
\hline \multirow[t]{3}{*}{ Total } & 52 & 8 & 60 & 52 & 8 & 60 \\
\hline & \multicolumn{3}{|c|}{ Chi-square $p=0.005$} & \multicolumn{3}{|c|}{ Chi-square $p=0.005$} \\
\hline & \multicolumn{3}{|c|}{ Fisher's Exact test $p=0.13$} & \multicolumn{3}{|c|}{ Fisher's Exact test $p=0.01 \mathrm{I}$} \\
\hline Caseinase + & 33 & 1 & 34 & 33 & I & 34 \\
\hline Caseinase - & 19 & 7 & 26 & 19 & 7 & 26 \\
\hline \multirow[t]{3}{*}{ Total } & 52 & 8 & 60 & 52 & 8 & 60 \\
\hline & \multicolumn{3}{|c|}{ Chi-square $P=0.0201$} & \multicolumn{3}{|c|}{ Chi-square $P=0.0201$} \\
\hline & \multicolumn{3}{|c|}{ Fisher's Exact test $p=0.0164$} & \multicolumn{3}{|c|}{ Fisher's Exact test $p=0.0164$} \\
\hline
\end{tabular}

Table 4 Variable Effects of fsr Genes on Gelatinase Activity

\begin{tabular}{|c|c|c|c|c|c|c|c|c|c|}
\hline & $f s r A+{ }^{a}$ & $f s r A-{ }^{a}$ & Total & $f s r B+{ }^{a}$ & $f s r B-{ }^{a}$ & Total & $f s r C+a$ & $f s r C-{ }^{a}$ & Total \\
\hline Gelatinase +ve & 12 & I & 13 & 12 & I & 13 & 12 & I & 13 \\
\hline Gelatinase -ve & 13 & 34 & 47 & 5 & 42 & 47 & 17 & 30 & 47 \\
\hline \multirow[t]{2}{*}{ Total } & 25 & 35 & 60 & 17 & 43 & 60 & 29 & 31 & 60 \\
\hline & \multicolumn{3}{|c|}{$\begin{array}{l}\text { Chi-square } p=0.00011 \\
\text { Fisher's Exact test } p=4 \times 10^{-5}\end{array}$} & \multicolumn{3}{|c|}{$\begin{array}{l}\text { Chi-square } p=5.453 \times 10^{-8} \\
\text { Fisher's Exact test } p=5 \times 10^{-8}\end{array}$} & \multicolumn{3}{|c|}{$\begin{array}{l}\text { Chi-square } p=0.00107 \\
\text { Fisher's Exact test } p=0.0004\end{array}$} \\
\hline
\end{tabular}

Note: ${ }^{a}$ These positive and negative signs reflect gene presence and absence (respectively), as determined by PCR.

gelatinase activity, consistent with prior findings. ${ }^{18,41}$ Other studies stated that expression of gelE is regulated by the $f_{s} r$ locus and does not merely depend on gelE, ${ }^{15,42}$ which is congruent with our findings here, as a significant and strong correlation between the presence of $f_{s r} A, f_{s r} B$, $f_{s r C}$ and the gelatinase activity was found. As in prior work from our laboratory, ${ }^{18} f_{s r} A$ or $f s r B$ can be a sufficient biomarker for gelatinase activity; however, $f_{s r} B$ remains the single strongest predictor (Figure 3 and Table 4) of that activity. 
The ability of $E$. faecalis to adhere to extracellular matrix proteins plays an important role in its pathogenesis. $^{2}$ The cell surface protein, Ace, is one of the microbial surface components recognizing adhesive matrix molecules (MSCRAMM) family that mediates the binding of $E$. faecalis to a certain type I collagen. ${ }^{43}$ All the isolates in the current study harbored the ace gene, and such high frequency in isolates from UTI was reported in other studies. ${ }^{30,33}$ Another factor associated with adherence is pili. Nallapareddy and colleagues reported that enterococci produce pili on their surface that help in bacterial adherence to host tissues and formation of biofilm. ${ }^{44}$ Here, the pil gene was present in all the isolates.

Aggregation substance on the bacterial surface helps bacteria to form large aggregates, a process believed to play a role in E. faecalis pathogenesis by facilitating the transfer of genetic material. ${ }^{2,45}$ High frequency of agg $(67 \%)$ was observed in the current study, agreeing with Seno et al's work $\left(82.7 \%\right.$ frequency in UTI samples) ${ }^{32}$ Of note, Kafil and Mobarez reported low frequency of agg gene in their enterococcal isolates from UTI ${ }^{30}$ Here we showed that agg was only significantly associated with the hemolysis phenotype, albeit this association was marginally significant (Fisher Exact test's $p=$ 0.043, but Chi-squared $p$ value was 0.065 ) - a result consistent with Chow et al's work on an enterococcal endocarditis model. ${ }^{46}$ The agg gene was neither associated with biofilm formation nor protease activities (Tables 3, $\underline{\mathrm{S} 2}$ and $\underline{\mathrm{S} 3}$ ). This finding may clarify the discrepant frequencies of this gene among UTI-isolated E. faecalis, as the gene does not seem to be associated with adhesion or biofilm formation, which are among the crucial virulence phenotypes for bacterial survival in the urinary tract. Moreover, Shankar and colleagues showed that the agg gene and cytolysin operon were clustered together on one pathogenicity island; ${ }^{47}$ yet, in our work, their presence was not strongly correlated (Figure 3) nor statistically significantly associated (Tables S2 and $\underline{\mathrm{S} 3}$ ).

Cytolysin is the toxin to which hemolysis has been primarily attributed. Although the $c y l$ gene was present in $54 \%$ of the isolates, only $33 \%$ of them showed blood hemolysis. However, this phenotype-genotype association was strongly statistically significant, as $c y l$ is a strong predictor of the activity; yet the gene obviously has to be expressed. Absence of blood hemolysis despite the presence of cyl was reported in other studies. ${ }^{48,49}$ Cytolysin expression was also found to be associated with increasing severity of infection. ${ }^{50}$
Of note, previous studies ${ }^{18,33,51}$ have analyzed virulence factor correlations in enterococci, in particular E. faecalis, isolated from Egyptian patients; however, the specificity of this work is that it solely focuses on E. faecalis samples from UTIs and that it calculates statistical associations between virulence genotypes and phenotypes, with particular emphasis on biofilm formation and adherence, in this type of clinically important infections.

Nonetheless, the study has a few limitations: The relatively small number of isolates allowed the identification of some statistical correlations, while other correlations could have been resolved with a higher number of isolates. Key virulence phenotypes were only tested in vitro, and the analysis was limited to UTIs to avoid redundancy with similar studies from the same geographical area. ${ }^{18,33,51}$

Future studies will address the current limitations and expand the study scope: As more specimens become available, the statistical will be revisited and extended to reexamine findings with marginal or low significance. Additionally, an in vivo model, eg, the wax moth Galleria mellonella, represents an attractive, affordable model for enterococcal virulence that can be used to bolster in vitro findings. Finally, wwhole-genome sequencing will allow more comprehensive, systems-level analysis of the virulence potential of the isolates (virulome analysis) as well as a comprehensive analysis of their resistance potential (resistome analysis).

\section{Conclusion}

The present study confirms the importance of adherence as an essential phenotype that characterizes enterococcal clinical isolates causing UTI, since all the isolates could form a biofilm. Although the strength of formed biofilms varied among the isolates, the majority formed moderate to strong biofilms in vitro. Besides biofilm formation, the isolates were also equipped with secreted toxins like proteases and cytolysin. Genotypic detection of virulence genes revealed a variable distribution of virulence genes among isolates, except for ace and pil genes, which are believed to contribute to the adherence of E. faecalis. Molecular detection of virulence genes also showed significant correlations between the presence of gelE and $s p r E$ genes and the strength of biofilm formed, and between $f_{s r} B$ and gelatinase activity, but confirmed prior findings that the presence of gelE is not sufficient to predict gelatinase activity, whereas the quorum sensing Fsr locus was an important predictor. Taken together, 
these results suggest $c y l$ as a biomarker for hemolytic activity, $f_{s r} B$ as a diagnostic biomarker for the gelatinase activity, and gelE-sprE as predictors for biofilm formation strength in E. faecalis.

\section{Ethics Approval}

The study has been approved by the ethical committees of both Faculty of Pharmacy, BUE and Cairo University. Samples were obtained from clinical laboratories, with no attached patient-identifying information.

\section{Disclosure}

The authors declare no financial or personal conflicts of interest related to this work.

\section{References}

1. Murray BE. The life and times of the Enterococcus. Clin Microbiol Rev. 1990;3(1):46-65. doi:10.1080/00472331003798640

2. Fisher K, Phillips C. The ecology, epidemiology and virulence of Enterococcus. Microbiology. 2009;155(6):1749-1757. doi:10.1099/ mic. $0.026385-0$

3. Poh CH, Oh HML, Tan AL. Epidemiology and clinical outcome of enterococcal bacteraemia in an acute care hospital. J Infect. 2006;52 (5):383-386. doi:10.1016/j.jinf.2005.07.011

4. Jett BD, Huycke MM, Gilmore MS. Virulence of enterococci. Clin Microbiol Rev. 1994;7(4):462-478. doi:10.1128/CMR.7.4.462

5. Ruoff KL, De La Maza L, Murtagh MJ, Spargo JD, Ferraro MJ. Species identities of enterococci isolated from clinical specimens. J Clin Microbiol. 1990;28(3):435-437. doi:10.1128/jcm.28.3.435437.1990

6. Kreft B, Marre R, Schramm U, Wirth R. Aggregation substance of Enterococcus faecalis mediates adhesion to cultured renal tubular cells. Infect Immun. 1992;60(1):25-30. doi:10.1128/IAI.60.1.2530.1992

7. Otto M. Targeted immunotherapy for staphylococcal infections. BioDrugs. 2008;22(1):27-36. doi:10.2165/00063030-20082201000003

8. Patti JM, Allen,BL, McGavin MJ, Hook M. MSCRAMM-mediated adherence of microorganisms to host tissues. Annu Rev Microbiol. 1994;48(1):585-617. doi:10.1146/annurev.micro.48.1.585

9. Donlan RM. Biofilms: microbial life on surfaces. Emerg Infect Dis. 2002;8(9):881-890. doi:10.3201/eid0809.020063

10. Mohamed JA, Huang DB. Biofilm formation by enterococci. $J$ Med Microbiol. 2007;56:1581-1588. doi:10.1099/jmm.0.47331-0

11. Ike Y, Hashimoto H, Clewell DB. Hemolysin of Streptococcus faecalis subspecies zymogenes contributes to virulence in mice. Infect Immun. 1984;45(2):528-530. doi:10.1128/iai.45.2.528-530.1984

12. Makinen PL, Clewell DB, An F, Makinen KK. Purification and substrate specificity of a strongly hydrophobic extracellular metalloendopeptidase ('gelatinase') from Streptococcus faecalis (strain 0G1-10). J Biol Chem. 1989;264(6):3325-3334. doi:10.1016/S00219258(18)94069-X

13. Franz CMAP, Huch M, Abriouel H, Holzapfel W, Gálvez A. Enterococci as probiotics and their implications in food safety. Int $J$ Food Microbiol. 2011;151(2):125-140. doi:10.1016/j. ijfoodmicro.2011.08.014

14. Hancock LE, Perego PM. The Enterococcus faecalis $f_{s} r$ two-component system controls biofilm development through production of gelatinase. $J$ Bacteriol. 2004;186(17):5629-5639. doi:10.1128/JB.186.17.5629-5639.2004
15. Qin X, Singh KV, Weinstock GM, Murray BE. Effects of Enterococcus faecalis $f_{s} r$ genes on production of gelatinase and a serine protease and virulence. Infect Immun. 2000;68 (5):2579-2586. doi:10.1128/iai.68.5.2579-2586.2000

16. Bose M, Chatterjee SS, Mukherjee K, et al. Enterococcal urinary tract infection: an emerging threat. J Evol Med Dent Sci. 2015;4 (17):2898-2904. doi:10.14260/jemds/2015/419

17. Kau AL, Martin SM, Lyon W, Hayes E, Caparon MG, Hultgren SJ. Enterococcus faecalis tropism for the kidneys in the urinary tract of C57BL/6J mice. Infect Immun. 2005;73(4):2461-2468. doi:10.1128/ IAI.73.4.2461-2468.2005

18. Hashem YA, Amin HM, Essam TM, Yassin AS, Aziz RK. Biofilm formation in enterococci: genotype-phenotype correlations and inhibition by vancomycin. Sci Rep. 2017;7(1). doi:10.1038/s41598-01705901-0

19. Christensen GD, Simpson WA, Younger JJ, et al. Adherence of coagulase-negative staphylococci to plastic tissue culture plates: a quantitative model for the adherence of staphylococci to medical devices. J Clin Microbiol. 1985;22(6):996-1006. doi:10.1128/ JCM.22.6.996-1006.1985

20. Marinho AR, Martins PD, Ditmer EM, et al. Biofilm formation on polystyrene under different temperatures by antibiotic resistant Enterococcus faecalis and Enterococcus faecium isolated from food. Brazilian J Microbiol. 2013;44(2):423-426. doi:10.1590/S151783822013005000045

21. Stepanović S, Vuković D, Dakić I, Savić B, Švabić-vlahović M. A modified microtiter-plate test for quantification of staphylococcal biofilm formation. J Microbiol Methods. 2000;40(2):175-179. doi:10.1016/S0167-7012(00)00122-6

22. Su YA, Sulavik MC, He P, et al. Nucleotide sequence of the gelatinase gene (gelE) from Enterococcus faecalis subsp. liquefaciens. Infect Immun. 1991;59(1):415-420. doi:10.1128/IAI.59.1.415420.1991

23. Frazier WC. A method for the detection of changes in gelatin due to bacteria. J Infect Dis. 1926;39(4):302-309. doi:10.1093/infdis/ 39.4.302

24. Archimbaud C, Shankar N, Forestier C, et al. In vitro adhesive properties and virulence factors of Enterococcus faecalis strains. Res Microbiol. 2002;153(2):75-80. doi:10.1016/S0923-2508(01) 01291-8

25. Furumura MT, Figueiredo PMS, Carbonell GV, Darini AL da C, Yano T. Virulence-associated characteristics of Enterococcus faecalis strains isolated from clinical sources. Brazilian J Microbiol. 2006;37 (3):230-236. doi:10.1590/S1517-83822006000300007

26. Dada AC, Ahmad A, Usup G, Heng LY, Hamid R. High-level aminoglycoside resistance and virulence characteristics among enterococci isolated from recreational beaches in Malaysia. Environ Monit Assess. 2013;185(9):7427-7443. doi:10.1007/ s10661-013-3110-x

27. Jasni AS, Mullany P, Hussain H, Roberts AP. Demonstration of conjugative transposon (Tn5397)-mediated horizontal gene transfer between Clostridium difficile and Enterococcus faecalis. Antimicrob Agents Chemother. 2010;54(11):4924-4926. doi:10.1128/ AAC.00496-10

28. Dutka-Malen S, Evers S, Courvalin P. Detection of glycopeptide resistance genotypes and identification to the species level of clinically relevant enterococci by PCR. J Clin Microbiol. 1995;33 (1):24-27. doi: $10.1128 / \mathrm{jcm} .33 .5 .1434-1434.1995$

29. Eaton TJ, Gasson MJ. Molecular screening of Enterococcus virulence determinants and potential for genetic exchange between food and medical isolates. Appl Environ Microbiol. 2001;67(4):1628-1635. doi:10.1128/AEM.67.4.1628

30. Kafil HS, Mobarez AM. Assessment of biofilm formation by enterococci isolates from urinary tract infections with different virulence profiles. J King Saud Univ - Sci. 2015;27(4):312-317. doi:10.1016/j. jksus.2014.12.007 
31. Lewis K. Riddle of biofilm resistance. Antimicrob Agents Chemother 2001;45(4):999-1007. doi:10.1128/AAC.45.4.999

32. Seno Y, Kariyama R, Mitsuhata R, Monden K, Kumon H. Clinical implications of biofilm formation by Enterococcus faecalis in the urinary tract. Acta Med Okayama. 2005;59(3):79-87. doi:10.18926/ AMO/31979

33. Shahi F, Hamidi H, Khoshnood S, Mehdipour G, Dezfouli A, Sheikh A. Virulence determinants and biofilm formation in clinical isolates of Enterococcus: a cross-sectional study. J Acute Dis. 2020;9 (1):27-32. doi:10.4103/2221-6189.276079

34. Soares RO, Fedi AC, Reiter KC, Caierão J, D'Azevedo PA. Correlation between biofilm formation and gelE, esp, and agg genes in Enterococcus spp. clinical isolates. Virulence. 2014;5 (5):634-637. doi:10.4161/viru.28998

35. Rahimi N, Poursina F, Sadat Ghaziasgar F, Sepehrpor S, Hassanzadeh A. Presence of virulence factor genes ( $g e l E$ and esp) and biofilm formation in clinical Enterococcus faecalis and Enterococcus faecium isolated from urinary tract infection in Isfahan, Iran. Gene Reports. 2018;13:72-75. doi:10.1016/j. genrep.2018.09.004

36. Toledo-arana A, Valle J, Solano C, Cucarella C, Lamata M, Amorena B. The enterococcal surface protein, Esp, is involved in Enterococcus faecalis biofilm formation. Appl Environ Microbiol. 2001;67(10):4538-4545. doi:10.1128/AEM.67.10.4538

37. Arciola CR, Baldassarri L, Campoccia D, et al. Strong biofilm production, antibiotic multi-resistance and high gelE expression in epidemic clones of Enterococcus faecalis from orthopaedic implant infections. Biomaterials. 2008;29(5):580-586. doi:10.1016/j. biomaterials.2007.10.008

38. Kart D, Kuştimur AS. Investigation of gelatinase gene expression and growth of Enterococcus faecalis clinical isolates in biofilm models. Turkish J Pharm Sci. 2019;16(3):356-361. doi:10.4274/tjps. galenos.2018.69783

39. Coque TM, Patterson JE, Steckelberg JM, Murray BE. Incidence of hemolysin, gelatinase, and aggregation substance among enterococci isolated from patients with endocarditis and other infections and from feces of hospitalized and community-based persons. J Infect Dis. 1995;171(5):1223-1229. doi:10.1093/infdis/171.5.1223

40. Sharifi Y, Hasani A, Ghotaslou R, et al. Virulence and antimicrobial resistance in enterococci isolated from urinary tract infections. $A d v$ Pharm Bull. 2013;3(1):197-201. doi:10.5681/apb.2013.032

41. Kashef M, Alvandi A, Hasanvand B, Azizi M, Abiri R. Virulence factor and biofilm formation in clinical enterococcal isolates of the west of Iran. Jundishapur J Microbiol. 2017;10(7). doi:10.5812/ jjm.14379
42. Nakayama J, Kariyama R, Kumon H. Description of a 23.9-kilobase chromosomal deletion containing a region encoding $f_{s} r$ genes which mainly determines the gelatinase-negative phenotype of clinical isolates of Enterococcus faecalis in urine. Appl Environ Microbiol. 2002;68(6):3152-3155. doi:10.1128/AEM.68.6.3152-3155.2002

43. Rich RL, Kreikemeyer B, Owens RT, et al. Ace is a collagen-binding MSCRAMM from Enterococcus faecalis. J Biol Chem. 1999;274 (38):26939-26945. doi:10.1074/jbc.274.38.26939

44. Nallapareddy SR, Singh KV, Sillanpää J, et al. Endocarditis and biofilm-associated pili of Enterococcus faecalis. J Clin Invest. 2006;116(10):2799-2807. doi:10.1172/JCI29021

45. Koch S, Hufnagel M, Theilacker C, Huebner J. Enterococcal infections: host response, therapeutic, and prophylactic possibilities. Vaccine. 2004;22(7):822-830. doi:10.1016/j.vaccine.2003.11.027

46. Chow JW, Thal LA, Perri MB, et al. Plasmid-associated hemolysin and aggregation substance production contribute to virulence in experimental enterococcal endocarditis. Antimicrob Agents Chemother. 1993;37(11):2474-2477. doi:10.1128/AAC.37.11.2474

47. Shankar N, Baghdayan AS, Gilmore MS. Modulation of virulence within a pathogenicity island in vancomycin-resistant Enterococcus faecalis. Nature. 2002;417(6890):746-750. doi:10.1038/nature00802

48. Kiruthiga A, Padmavathy K, Shabana P, Naveenkumar V, Gnanadesikan S, Malaiyan J. Improved detection of esp, hyl, asal, gelE, cylA virulence genes among clinical isolates of enterococci. BMC Res Notes. 2020;13(1):1-7. doi:10.1186/s13104-020-05018-0

49. Hällgren A, Claesson C, Saeedi B, Monstein HJ, Hanberger H, Nilsson LE. Molecular detection of aggregation substance, enterococcal surface protein, and cytolysin genes and in vitro adhesion to urinary catheters of Enterococcus faecalis and E. faecium of clinical origin. Int J Med Microbiol. 2009;299(5):323-332. doi:10.1016/j. ijmm.2008.10.001

50. Van Tyne D, Martin MJ, Gilmore MS. Structure, function, and biology of the Enterococcus faecalis cytolysin. Toxins. 2013;5 (5):895-911. doi:10.3390/toxins5050895

51. Tawfick MM, El Menofy NG, Omran ME, Alsharony OA, AboShady MA. Phenotypic and molecular characterization of plasmidmediated virulence and antimicrobial resistance traits among multidrug resistant Enterococcus spp. in Egypt. J Pure Appl Microbiol. 2020;14(3):1649-1661. doi:10.22207/JPAM.14.3.03
Infection and Drug Resistance

\section{Publish your work in this journal}

Infection and Drug Resistance is an international, peer-reviewed openaccess journal that focuses on the optimal treatment of infection (bacterial, fungal and viral) and the development and institution of preventive strategies to minimize the development and spread of resistance. The journal is specifically concerned with the epidemiology of antibiotic resistance and the mechanisms of resistance development and diffusion in both hospitals and the community. The manuscript management system is completely online and includes a very quick and fair peerreview system, which is all easy to use. Visit http://www.dovepress.com/ testimonials.php to read real quotes from published authors. 